\title{
Assistência de enfermagem e dor em pacientes ortopédicos na recuperação anestésica, no Brasil *
}

\author{
Nursing assistance and pain in orthopedic patients during anesthetic recovery in Brazil \\ Gisele Reis de Paula ${ }^{1}$, Vanda dos Santos Reis ${ }^{1}$, Flávia Alves Ribeiro², Maria Teresa Gagliazzi ${ }^{3}$ \\ * Recebido do Curso de Graduação em Enfermagem da Universidade de Mogi das Cruzes (UMC). Mogi das \\ Cruzes, SP.
}

\section{RESUMO}

JUSTIFICATIVA E OBJETIVOS: A dor traz prejuízos na recuperação do paciente submetido à cirurgia ortopédica. O objetivo deste estudo foi descrever as informações relacionadas à experiência dolorosa de pacientes em pós-operatório de cirurgias ortopédicas na sala de recuperação pós-anestésica (SRPA) que podem contribuir para a assistência de Enfermagem.

CONTEÚDO: Trata-se de estudo com abordagem qualitativa, as fontes para a coleta das informações foram as bases de dados da plataforma da BIREME, os objetos foram artigos que atenderam aos seguintes critérios de inclusão previamente estabelecidos, a coleta ocorreu mediante busca on-line, a abordagem das informações foi realizada pelo método da Hermenêutica Dialética. Após esta abordagem, as categorias levantadas foram: "O uso da anestesia regional diminui a intensidade da dor pós-operatória em cirurgia ortopédica", "Terapias para o tratamento da dor pós-operatória em cirurgia ortopédica", "A avaliação auxilia no gerenciamento da dor no pós-operatório de cirurgia ortopédica”.

CONCLUSÃO: O controle da dor pós-operatória é importante em pacientes ortopédicos e a anestesia regional pode

1. Aluna do Curso de Enfermagem da Universidade de Mogi das Cruzes (UMC). Mogi das Cruzes, SP, Brasil.

1. Enfermeira Especialista em Enfermagem Médico Cirúrgica. Mestranda em Enfermagem. Docente do Curso de Enfermagem da Universidade de Mogi das Cruzes (UMC). Mogi das Cruzes, SP, Brasil.

3. Enfermeira Mestre em Enfermagem. Coordenadora do Curso de Enfermagem da Universidade de Mogi das Cruzes (UMC). Mogi das Cruzes, SP, Brasil.

Endereço para correspondência:

Flávia Alves Ribeiro

Rua José de Jesus, 66/54 - Morumbi

05630-090 São Paulo, SP.

Fones: (11) 9440-9146 - (11) 7417-1878.

E-mail: flaviaalvesribeiro@hotmail.com proporcionar a analgesia preventiva para o pós-operatório imediato. $\mathrm{O}$ enfermeiro deve estabelecer o diagnóstico de enfermagem e as intervenções devem ser precedidas pela avaliação da intensidade, da qualidade e dos fatores que interferem na dor experimentada pelo paciente ortopédico.

Descritores: Cirurgia ortopédica, Dor aguda, Enfermagem perioperatória, Sala de recuperação.

\section{SUMMARY}

BACKGROUND AND OBJECTIVES: Pain is noxious for the recovery of patients submitted to orthopedic surgery. This study aimed at describing information on the pain experience of patients in the postoperative period of orthopedic surgeries in the post-anesthetic recovery unit (PACU), which may be useful for the Nursing assistance.

CONTENTS: This is a qualitative study using BIREME's platform databases to collect information. The aims were articles meeting the following and previously established inclusion criteria and data were collected by online search. Information was approached by the Dialectic Hermeneutics method. After this approach, the following categories were surveyed: "Regional anesthesia decreases postoperative pain in orthopedic surgeries", "Therapy for postoperative pain of orthopedic surgeries", "Evaluation helps pain management in the postoperative period of orthopedic surgeries".

CONCLUSION: Postoperative pain control is important for orthopedic patients and regional anesthesia may provide preventive analgesia for the immediate postoperative period. The nursing team shall establish the nursing diagnosis and interventions shall be preceded by the evaluation of intensity, quality and factors interfering with pain felt by orthopedic patients.

Keywords: Acute pain, Orthopedic surgery, Perioperative nursing, Recovery room. 


\section{INTRODUÇÃO}

A dor traz prejuízos na recuperação do paciente submetido à cirurgia ortopédica, os quais estão relacionados a alterações fisiológicas como a demora em deambular e o risco de tromboembolismo pela imobilidade e alterações emocionais, pelo distanciamento da família e autoimagem alterada.

A dor pós-operatória é relatada por número significativo de pacientes como a pior experiência da sua vida. Associam-se à dor fatores de complicações físicas e emocionais que podem agravar a situação de doença ou trauma que motivou a cirurgia ${ }^{1-3}$.

No pós-operatório imediato, o desconforto doloroso pode alterar o metabolismo do paciente, afetando os sistemas pulmonar, cardiovascular, gastrintestinal, urinário, neurológico e endócrino. Seu alívio traz, portanto, diminuição destas intercorrências ${ }^{4,5}$.

$\mathrm{O}$ gerenciamento adequado da dor pós-operatória na sala de recuperação pós-anestésica (SRPA), no caso do paciente ortopédico, controla o seu estresse físico e psicológico, além de permitir a elaboração de cuidados relacionados à mobilidade e à movimentação ${ }^{5}$.

Em se tratando de pacientes ortopédicos, as ferramentas para o gerenciamento da dor são as farmacológicas (administração de medicamentos), e não farmacológicas (mobilidade e movimentação, acupuntura, massagem terapêutica, relaxamento, posicionamento e conforto).

Dentre as alternativas para analgesia farmacológica no pós-operatório de cirurgia ortopédica destacam-se a infusão de analgésico por via peridural, a analgesia controlada pelo paciente (ACP) e a administração por via venosa de medicação; além disso, as intervenções de Enfermagem podem representar algumas das medidas não farmacológicas de controle e melhoria dos quadros dolorosos ${ }^{3,6,7}$.

O papel da Enfermagem no controle da dor pós-operatória do paciente ortopédico consiste na avaliação da intensidade e na adoção de estratégias para minimizar o desconforto, mediante o planejamento dos cuidados, levando em consideração as alterações dos sinais vitais, das condições físicas e emocionais e do quadro doloroso propriamente dito.

O enfermeiro é um elemento importante para cuidar de maneira segura dos pacientes que vivenciam complicações no pós-operatório imediato, dentre estas complicações, destaca-se a dor ${ }^{8}$.

A área de Enfermagem no controle da dor passa por constantes avanços, pelo desenvolvimento de técnicas e equipamentos para analgesia; deste fato emergiu a necessidade de atualização permanente dos profissionais ${ }^{3}$. O enfermeiro tem competências relacionadas ao uso de outras terapias além das farmacológicas para minimizar a dor dos pacientes e contribuir para a expectativa de restabelecimento, dentre estas terapias, destacam-se a musicoterapia, a massagem terapêutica, a estimulação de pensamentos que aliviem a dor, a aromaterapia e o posicionamento para conforto?.

A equipe de Enfermagem deve ter uma perspectiva ampla sobre as sensações experimentadas e relatadas pelos pacientes com dor no pós-operatório imediato porque permanece a maior parte do tempo da internação prestando assistência; entretanto, relatam insatisfação com o gerenciamento da sua dor pela Enfermagem².

$\mathrm{O}$ fato de grande número de pacientes que sofreram cirurgia ortopédica para amputação de membros e experimentaram a cronificação da dor aguda pós-operatória por negligência no gerenciamento desta, suscita necessidade de esforços da equipe de saúde no sentido de melhorar as práticas assistenciais para estes pacientes ${ }^{6}$.

A partir da reflexão sobre os relatos desses autores, emergiu a questão desta pesquisa: "Quais são as informações relacionadas à experiência dolorosa de pacientes em pós-operatório de cirurgias ortopédicas na SRPA que podem contribuir para a assistência de Enfermagem?" Justifica-se a realização deste estudo baseadas na intenção de contribuir para diminuição do sofrimento causado pela dor aguda pós-operatória em pacientes ortopédicos, de melhorar o cuidado prestado pelos profissionais de Enfermagem a partir das informações já explicitadas. O presente estudo teve como objetivo descrever as informações levantadas na revisão bibliográfica relacionadas à experiência dolorosa de pacientes em pós-operatório de cirurgias ortopédicas na SRPA que podem contribuir para a assistência de Enfermagem.

\section{CONTEÚDO METODOLÓGICO}

Após leitura exaustiva dos artigos selecionados frente à questão norteadora foram elaboradas as seguintes categorias: "O uso da anestesia regional diminui a intensidade da dor pós-operatória em cirurgia ortopédica", "Terapias para o tratamento da dor pós-operatória em cirurgia ortopédica", "A avaliação auxilia no gerenciamento da dor no pós-operatório de cirurgia ortopédica”.

\section{O uso da anestesia regional diminui a intensidade da dor pós-operatória em cirurgia ortopédica}

Esta categoria emergiu dos contextos expostos pelos autores pesquisados relacionando a eficácia da anestesia regional para o controle da dor de pacientes submetidos à cirurgia ortopédica, transcritos a seguir:

"O bloqueio [... melhorou de maneira significativa a qualidade da analgesia pós-operatória da artroplastia total de joelho...]"10; 
"O bloqueio intra-articular traz ótimos resultados aos pacientes submetidos à cirurgia artroscópica de joelho" "11; "A anestesia regional proporcionou melhor analgesia", "[... o bloqueio é efetivo para analgesia pós-operatória nos pacientes submetidos às cirurgias ortopédicas de quadril, fêmur ou joelho...] "12.

"O entendimento da extensão dos bloqueios... [proporciona uma analgesia de melhor qualidade nos procedimentos cirúrgicos de quadril e fêmur"' ${ }^{13}$;

Percebe-se, por essas expressões que os autores acreditam serem os bloqueios de nervos periféricos a mais eficaz opção para procedimentos ortopédicos, notadamente, nos de membros inferiores.

O conhecimento dessas constatações tem influência para a assistência de Enfermagem no sentido de que o enfermeiro, quando ciente do procedimento anestésico e do tipo do bloqueio executado, espera, após a cirurgia, um período de recuperação mais tranquilo no tocante ao paciente não experimentar a dor.

Em estudo retrospectivo sobre as intervenções do enfermeiro e as complicações em SRPA ${ }^{8}$ de 216 pacientes sob anestesia geral em até $54 \%$ a dor foi uma complicação prevalente e o enfermeiro deste setor, executou uma assistência diferenciada, considerando tal prevalência. As afirmações expressas ${ }^{8}$ remetem à reflexão de que a assistência de Enfermagem ao paciente ortopédico com dor na SRPA é permeada pela ciência e consequente entendimento acerca da técnica anestésica utilizada.

Essa assertiva anterior encontra ressonância na expressão que valoriza outro tipo de técnica anestésica, a regional, como se lê:

“[... pacientes ortopédicos submetidos à anestesia regional não apresentam complicações relacionadas à dor no pós-operatório...] [... o que direciona a assistência a estes pacientes...]" "I2;

"Este conhecimento (de que a anestesia regional proporciona melhor analgesia) é importante para prevenir o intenso catabolismo que a dor pode desencadear".

$\mathrm{O}$ enfermeiro, para o gerenciamento da dor necessita, portanto, de competências relacionadas ao conhecimento da anestesia, suas técnicas, fármacos utilizados e efeitos colaterais esperados, que em contrapartida, se vê alertado:

"A dor pós-operatória (em pacientes ortopédicos) tem sido bastante investigada, porém, é preciso analisar se a terapêutica analgésica empregada é eficaz"4.

Portanto, as reflexões dialéticas que podem emergir dos prós e contras relativos às técnicas analgésicas e anestésicas para cirurgias ortopédicas contribuem para o aprofundamento e aprimoramento desses conhecimentos específicos que o enfermeiro deve adquirir e compartilhar para colocar em prática um cuidado adequado aos pacientes na SRPA, considerados críticos ${ }^{8}$.

Emerge, portanto, desta categoria, que pacientes submetidos à anestesia regional em quadril, fêmur ou joelho experimentam menos dor na SRPA do que àqueles submetido à anestesia geral, e o enfermeiro conhecedor de tal evidência, deve refletir e discernir direcionando com maior competência seu cuidado.

\section{Terapias para o tratamento da dor pós-operatória em cirurgia ortopédica}

Os estudos que deram origem a esta categoria abordaram as maneiras disponíveis para tratar a dor do paciente ortopédico na SRPA, quando esta, ou necessita prevenção ou já está instalada, algumas expressões a seguir, exemplificam a categoria em questão:

"O tratamento da dor pós-operatória objetiva, além do simples alivio, a prevenção das complicações próprias da dor" 14

"A descoberta de receptores opioides na medula trouxe novas perspectivas para o campo, da analgesia, com a possibilidade de uso dos opioides diretamente na raquianestesia"14,

"As terapias complementares baseiam-se em promover alivio da dor","

"O tratamento inadequado da dor pós-operatória resulta em aumento da morbimortalidade",

"A morfina é o analgésico opioide de primeira escolha no tratamento da dor aguda de grande intensidade, como é a dor pós-operatória";

"É possivel e importante o atendimento aos pacientes no período pós-operatório com relação ao tratamento da dor aguda" 6 .

O tratamento da dor pós-operatória em pacientes submetidos à cirurgia ortopédica consiste no uso associado de terapias complementares e farmacológicas:

"As terapias complementares como adjuvantes ao tratamento farmacológico proporcionando o alivio da dor e do sofrimento proporcionado pelo quadro doloroso" . Dentre as alternativas chamadas "complementares" para o alívio da dor, destaca-se o toque, o uso da música e de aromas, além da estimulação da imaginação do paciente: "As principais terapias complementares para alívio do quadro doloroso são técnicas de relaxamento, estimulação cutânea, a aromaterapia, a imaginação guiada, a terapia vibracional e a música".

O enfermeiro age no suprimento das necessidades emocionais e físicas do paciente com dor, nos aspectos emocionais e subjetivos atua de maneira não farmacológica e no campo biológico, usa técnicas farmacológicas para alívio ${ }^{8}$. 
Percebe-se que, quando aplicadas adequadamente em seus objetivos, as práticas medicamentosas e complementares associadas trazem resultados positivos em relação ao conforto e tratamento da dor.

"O contato constante do enfermeiro com o paciente favorece a implementação de terapias que aliviem a dor, promovendo assistência integralizada e melhora da qualidade de vida do paciente com dor"'.

Em outra vertente, há de se ressaltar a importância do tratamento farmacológico para o alívio da dor pós-operatória em Ortopedia, como expressam alguns autores:

"Para o tratamento da dor pós-operatória têm sido empregados analgésicos não opioides, anti-inflamatórios não esteroides que, eventualmente, são associados à opioides fracos" "5;

"A qualidade da analgesia obtida pelo uso de morfina no alivio da dor pós-operatória de pacientes submetidos à cirurgia ortopédica foi satisfatória" "14;

"Em nossa prática, observamos que 4\% dos pacientes submetidos à cirurgia ortopédica requerem tratamento farmacológico da dor"'6.

Além dos aspectos relacionados à terapia complementar, o enfermeiro precisa estar atento aos benefícios físicos que a terapia farmacológica pode trazer em relação à prevenção da cronificação da dor e da mobilização precoce nos pacientes ortopédicos ${ }^{8}$.

Emerge, portanto, a reflexão acerca dos dados desta categoria que terapias complementares e farmacológicas associadas ou não, são eficazes quando utilizadas de maneira a responder às necessidades básicas afetadas pelos pacientes ortopédicos, tanto do ponto de vista emocional quanto do fisiológico, em torno deste, ressalta-se a importância dos protocolos medicamentosos disponíveis e em estudo relacionados à dor aguda em Ortopedia.

\section{A avaliação auxilia no gerenciamento da dor no pós- -operatório de cirurgia ortopédica}

Esta categoria emergiu do entendimento dos autores dos artigos lidos sobre a importância da avaliação da dor que o paciente ortopédico sente como se observa a seguir:

"Esta tarefa (de avaliação da dor e administração de analgésico... é de extrema importância" "7;

"Os profissionais da saúde precisam conhecer os métodos utilizados para a avaliação da dor"”;

"Enfatizamos a necessidade... de mensuração da dor no meio cirúrgico" 15.

Estas expressões claramente registram a importância da avaliação da dor e que não depende unicamente dos instrumentos disponíveis para o uso, mas associados ao preparo holístico do profissional de Enfermagem para avaliar a dor no pós-operatório, neste caso, de cirurgia ortopédica, mes- mo parecendo um desafio de controle desse sinal. Esta assertiva encontra congruências nas expressões dos autores:

"Mensurar a dor tem sido um desafio para aqueles que almejam controlar adequadamente tão complexa experiência" 15 ;

"Os enfermeiros devem esforçar-se para proteger o paciente de complicações".

Os instrumentos de avaliação da dor no pós-operatório de cirurgia ortopédica avaliam de maneira unidimensional ou em múltiplas dimensões a experiência dolorosa, que podem ser verificados pelo que segue:

"Dentre eles, destacam-se as escalas numéricas, nas quais se utilizam categorias numéricas; as escalas verbais, nas quais se utilizam categorias adjetivas, e as escalas analógicas visuais, nas quais há possibilidade de julgamentos visuais numa dimensão tomada como padrão"15.

Também, é imperioso destacar os instrumentos da enfermagem como uma forma associada de avaliar a dor no pós-operatório de cirurgia ortopédica que são as características definidoras do Diagnóstico de Enfermagem "Dor", assim registrados: "Os diagnósticos de Enfermagem são identificados a partir de características definidoras, fatores e situações iminentes de risco" 16 .

Na sensibilização do profissional para avaliar a dor, são necessários não só instrumentos de mensuração da intensidade da dor, mas também, os fatores associados, que o enfermeiro deve considerar para sua intervenção, como se verifica:

"Identificar a existência de queixa álgica e estabelecer a etiologia do sintoma, caracterizar a experiência dolorosa em todos os seus domínios, aferir as repercussões da dor no funcionamento biológico, emocional e comportamental do individuo"'s.

Para o alcance deste cuidado complexo, holístico e abrangente são necessárias estratégias de educação continuada da equipe de Enfermagem que atende pacientes em pós-operatório, especificamente neste caso, de cirurgia ortopédica:

"[... o enfermeiro deve empenhar-se na realização de programas de capacitação da equipe de Enfermagem que visem o esclarecimento de formas de identificação da dor] "16. Esta declaração remete à reflexão da importância do enfermeiro ser exclusivamente dedicado à SRPA, o que muitas vezes não ocorre, dividindo suas responsabilidades com outras atividades do bloco cirúrgico ou administrativas gerais. Esta multiplicidade de responsabilidades o impede de identificar e estimular a sua equipe a avaliar constantemente a dor dos pacientes em pós-operatório imediato. Ao contrário, se exclusivo na SRPA, pode implementar estratégias para destacar a importância da mensuração da dor e implementá-la prevenindo a cronificação álgica ${ }^{8}$.

Entende-se que a avaliação da dor no pós-operatório imediato de cirurgia ortopédica é permeada pelo uso de 
instrumentos validados agregados à assistência holística do enfermeiro, consideração dos fatores associados e capacitação da equipe de Enfermagem.

Por meio das informações coletadas pode-se pensar a importância do controle da dor pós-operatória em pacientes ortopédicos e evidenciar que a anestesia regional pode ser fonte de analgesia preventiva para o pós-operatório imediato, as terapias para tratamento da dor instaladas podem ser farmacológicas ou não farmacológicas e devem ser consideradas pelo enfermeiro para estabelecer seu diagnóstico de enfermagem e intervenções precedidas da avaliação da intensidade, da qualidade e de fatores que interferem na ocorrência da dor experimentada pelo paciente ortopédico.

De posse destas informações, sugere-se, em estudos futuros, a elaboração de protocolos de cuidados de Enfermagem a pacientes em pós-operatório de cirurgia ortopédica na SRPA embasados nestas premissas.

\section{CONCLUSÃO}

O controle da dor pós-operatória é importante em pacientes ortopédicos e a anestesia regional pode proporcionar a analgesia preventiva para o pós-operatório imediato. A terapia para tratamento da dor pode ser farmacológica ou não farmacológica e o enfermeiro deve estabelecer o diagnóstico de Enfermagem e as intervenções devem ser precedidas pela avaliação da intensidade, da qualidade e dos fatores que interferem na dor experimentada pelo paciente ortopédico.

\section{REFERÊNCIAS}

1 Charlton EJ, (editor). Core curriculum for professional education in pain. $3^{\text {nd }}$ ed: IASP Press; 2005.

2. Rocha LS, Moraes MW. Assistência de Enfermagem no controle da dor da Sala de Recuperação Anestésica. Rev Dor 2010;11(3):254-8.

3. Pimenta CAM, Santos EMM, Chaves LD, et al. Controle da dor no pós-operatório. Rev Esc Enferm USP 2001;35(2):180-3.

4. Landgraf CS, Marques RC, Pires OC, et al. Avaliação da analgesia pós-operatória em um hospital universitário. Rev Dor 2010;11(4):319-22.

5. Custódio G, Zappelini CEM, Trevisol DJ, et al. Uso de analgésicos nos pós-operatório para tratamento da dor em hospital no Sul do Brasil. Arq Catarinenses de Medicina 2008;37(4):75-9.

6. Valverde Filho J, Ruiz Neto PP, Freire RCMC, et al. Análise descritiva de serviço de dor aguda pós-operatória em hospital terciário. Rev Bras Anestesiol 2000;50(5):386-90.
7. Tacla MTGM, Hayashida M, Lima RAG. Registros sobre dor pós-operatória em crianças: uma análise retrospectiva de hospitais de Londrina, PR, Brasil. Rev Bras Enferm 2008;61(13):289-95.

8. Popov DCS, Peniche ACG. As intervenções do enfermeiro e as complicações em sala de recuperação pós-anestésica. Rev Esc Enferm USP 2009;43(4):953-61.

9. Eler GJ, Jaques AE. O enfermeiro e as terapias complementares para o alívio da dor. Arq Ciênc Saúde 2006;10(3):185-90.

10. Zugliane AH, Verçosa N, Amaral JLG, et al. Controle da dor pós-operatória da artroplastia total do joelho: é necessário associar o bloqueio do nervo isquiático ao bloqueio do nervo femoral? Rev Bras Anestesiol 2007;57(5):514-24.

11. Lopes MB, Sousa LR, Porsani DF, et al. Associação de bupivacaína e morfina intra-articular para analgesia pós-operatória em cirurgia artroscópica de joelho. Rev Bras Anestesiol 1999;49(3):165-8.

12. Fonseca NM, Ruzi RA, Ferreira FX, et al. Analgesia pós-operatória em cirurgia ortopédica. Estudo comparativo entre o bloqueio do plexo lombar por via perivascular inguinal (3 em 1) com ropivacaína e a analgesia subaracnóidea com morfina. Rev Bras Anestesiol 2003;53(2):188-97.

13. Imbelloni LE, Beato L, Beato C, et al. Analgesia pós-operatória para procedimentos cirúrgicos ortopédicos de quadril e fêmur: comparação entre bloqueio do compartimento do psoas e bloqueio perivascular inguinal. Rev Bras Anestesiol 2008;56(6):619-29.

14. Martelete M, Fiori AMC, Oliveira MLV. Analgesia pós-operatória com morfina peridural em pacientes submetidos à cirurgia de membros inferiores. Rev Bras Anestesiol 1998;38(3):189-92.

15. Pereira LV, Sousa FAEF. Mensuração e avaliação da dor pós-operatória: uma breve revisão. Rev Lat Am Enfermagem 1998;6(3):77-84.

16. Silva FS, Viana MF, Volpato MP. Diagnósticos de enfermagem em pacientes internados pela clínica ortopédica em unidade médico-cirúrgica. Rev Gaúcha Enferm 2008;29(4):565-72.

17. Pimenta CAM, Santos EMM, Chaves LD, et al. Controle da dor no pós-operatório. Rev. Esc Enferm USP 2001;35(2):180-3.

18. Rocha LS, Moraes MW. Assistência de enfermagem no controle da dor da sala de recuperação anestésica. Rev Dor 2010;11(3):254-8.

Apresentado em 15 de junho de 2011.

Aceito para publicação em 24 de agosto de 2011. 